\title{
Hipomineralização molar-incisivo: etiologia, características clínicas e tratamento
}

\author{
Molar incisor hypomineralization: etiology, clinical characteristics and treatment
}

\author{
Lunna Farias $^{1 *}$, Isla Camilla Carvalho Laureano ${ }^{1}$, Catarina Ribeiro Barros de Alencar², Alessandro Leite \\ Cavalcanti ${ }^{3}$
}

${ }^{1}$ Mestranda em Clínica Odontológica pelo Programa de Pós-Graduação em Odontologia da Universidade Estadual da Paraiba.; ${ }^{2}$ Pós-doutoranda do Programa de Pós-Graduação em Odontologia da Universidade Estadual.; ${ }^{3}$ Professor

Doutor do Departamento de Odontologia da Universidade Estadual da Paraíba.

\begin{abstract}
Resumo
Introdução: a Hipomineralização Molar-Incisivo (HMI) é um defeito de desenvolvimento do esmalte dentário que afeta de um até todos os primeiros molares permanentes, podendo estar presente nos incisivos permanentes. Objetivo: revisar a literatura sobre a HMI objetivando proporcionar informações ao cirurgião-dentista que realiza atendimento infantil sobre o diagnóstico, características clínicas e tratamento. Metodologia: revisão integrativa da literatura, sendo utilizado as bases de dados LILACS, PubMed, Web of Science e Scopus. Foram usadas as palavras-chaves “Hipoplasia do Esmalte Dentário/Dental Enamel Hypoplasia” e "Hipomineralização Molar-Incisivo/Molar Incisor Hypomineralization". Resultados: fatores etiológicos associados a complicações pré, peri e pós-natais, ambientais e genéticos têm sido evidenciados e a prevalência de HMI mostra-se variada entre as populações. Apresenta-se como manchas opacas bem delimitadas e, com o aumento da gravidade, ocorre a perda do esmalte resultando em fratura, destruição coronária e indicação de exodontia. As fraturas pós-eruptivas atuam como áreas de retenção de biofilme, o que facilita seu acúmulo e aumenta a susceptibilidade à cárie dentária. A Academia Europeia de Odontopediatria (EAPD) estabeleceu critérios que facilitam o diagnóstico deste agravo por meio de pesquisas epidemiológicas. As medidas terapêuticas são direcionadas de acordo com a gravidade da lesão. Conclusão: a HMI deve receber adequada atenção do cirurgião-dentista em função das consequências clínicas e estéticas que acarreta ao paciente, interferindo negativamente na sua qualidade de vida nos casos mais graves. Novas pesquisas são necessárias com a finalidade de expandir o conhecimento acerca da $\mathrm{HMI}$, objetivando ofertar ao paciente o correto diagnóstico e a melhor opção terapêutica.

Palavras-chave: Hipoplasia do Esmalte Dentário. Odontopediatria. Criança. Cárie Dentária.
\end{abstract}

\begin{abstract}
Introduction: molar Incisor Hypomineralization (HMI) is a developmental defects of enamel that affects from one to all first permanent molars and also permanent incisors. Aim: to review the literature with the aim of providing information to the pediatric dentist about the diagnosis, clinical characteristics and treatment of HMI. Methodology: integrative literature review, using the LILACS, PubMed, Web of Science and Scopus databases. The keywords "Hipoplasia do Esmalte Dentário/Dental Enamel Hypoplasia" and "Hipomineralização Molar-Incisivo/Molar Incisor Hypomineralization" were used. Results: etiologic factors are associated with prenatal and postnatal factors, environmental and genetic complications. The prevalence of HMI is varied among the populations. It's clinically characterized as delimited opaque spots. With increasing severity, can occurs enamel loss, fracture, coronary destruction and indication of exodontia. Post-eruptive fractures act as areas of biofilm retention, which facilitates their accumulation and increases susceptibility to dental caries. European Academy of Pediatric Dentistry (EAPD) has established criteria that facilitate diagnosis of $H M I$ in epidemiological research. Therapeutic measures are indicated according to the severity of the lesion. Conclusion: MIH should receive adequate attention from the dentist due to its clinical and aesthetic consequences, interfering negatively in quality of life of patients in the most severe cases. New research is needed to expand knowledge, aiming to offer the patient the correct diagnosis and the best therapeutic option.
\end{abstract}

Keywords: Dental Enamel Hypoplasia. Pediatric Dentistry. Child. Dental Caries.

\section{INTRODUÇÃO}

Os defeitos de desenvolvimento do esmalte são anormalidades frequentemente observadas na dentição humana decídua ou permanente, os quais podem se classificar como hipoplasia ou hipomineralização do órgão dentário (ELFRINK et al., 2012). Ambos são causados por

Correspondente/Corresponding: *Lunna Farias - End: Rua Frei Caneca, 275, apto 101, Centro - Campina Grande, PB CEP: 58400-295 - Tel: falta -E-mail: lunna_farias@hotmail.com múltiplos fatores, que vão desde condições genéticas à fatores ambientais, incluindo condições sistêmicas adquiridas, como desnutrição, doenças comuns na infância (catapora, otite média, infecções respiratórias e do trato urinário) e uso de antibióticos (FORD et al., 2009; SEOW, 2014; WHATLING; FEARNE, 2008).

A hipoplasia é um defeito resultante da alteração dos ameloblastos durante a formação da matriz dentária provocando a redução da quantidade de esmalte (CLARKSON, 1992; ELFRINK et al., 2012; SEOW, 2014). A hipominerali- 
zação, por sua vez, se configura como uma deficiência na qualidade do esmalte e decorre de um distúrbio durante o momento de calcificação ou maturação, apresentando clinicamente translucência anormal e opacidade do esmalte (ELFRINK et al., 2012; GUERGOLETTE et al., 2009; YANNAM; AMARLAL; REKHA, 2016).

Dentre essas alterações, destaca-se a Hipomineralização Molar-Incisivo (HMI) que afeta um ou até todos os primeiros molares permanentes, podendo estar presente também nos incisivos permanentes (ANDRADE et al., 2017; TEIXEIRA et al., 2017; WEERHEIJM et al., 2003). $\mathrm{Na}$ literatura odontológica, diversos termos podem ser encontrados identificando esta condição, como: opacidades de esmalte não-fluoróticas, manchas opacas, opacidades de esmalte idiopático e molares de queijo (SUBRAMANIAM; GUPTA; SHARMA, 2016; WEERHEIJM; JALEVIK; ALALUUSUA, 2001). Apenas em 2001, foi sugerido o uso unificado do termo atual - Hipomineralização Molar-Incisivo (WEERHEIJM; JALEVIK; ALALUUSUA, 2001).

A HMI apresenta-se como um grande desafio clínico para o cirurgião-dentista (TEIXEIRA et al., 2017), uma vez que as alterações na estrutura do elemento dentário acarreta inúmeras consequências clínicas dentre as quais podem ser citadas: fraturas pós-eruptivas do esmalte (AHMADI; RAMAZANI; NOURINASAB, 2012; LYGIDAKIS et al., 2010), maior susceptibilidade à lesão cariosa (AHMADI; RAMAZANI; NOURINASAB, 2012; LYGIDAKIS et al., 2010), hipersensibilidade dentária (BUCHGRABER; KQIKU; EBELESEDER, 2017), adesão deficiente do material restaurador ao esmalte dentário (JASULAITYTE; VEERKAMP; WEERHEIJM, 2007), necessidade de retratamento com frequência (SALEM; AZIZ; ASADI, 2016; WEERHEIJM, 2004), envolvimento dos fatores psicológicos do paciente e seu núcleo familiar como maior ansiedade odontológica (ALLAZZAM; ALAKI; EL MELIGY, 2014; KOSMA et al., 2016) e o impacto negativo na qualidade de vida (DANTAS-NETA et al., 2016). Além disso, os pacientes relatam problemas estéticos quando os incisivos são afetados (TOURINO et al., 2016; WEERHEIJM, 2003).

O diagnóstico precoce e os cuidados preventivos são essenciais para o sucesso do tratamento dos defeitos de desenvolvimento do esmalte (SEOW, 2014). Além disso, como a formação do esmalte dos molares e incisivos permanentes ocorre ao mesmo tempo que os molares decíduos, a presença de defeitos de esmalte nestes indica um risco para que os defeitos ocorram na dentição permanente (SEOW, 2014; SEOW et al., 2011). Deste modo, crianças com HMI em molares decíduos devem ter os dentes permanentes monitorados quanto à presença de defeitos semelhantes (SEOW, 2014). Portanto, é importante que o cirurgião-dentista tenha um adequado conhecimento sobre esta condição e esteja devidamente preparado para o manejo do paciente pediátrico, como também saiba diferenciar essa patologia de outras alterações do esmalte dentário (VILANI et al., 2014).

Frente ao exposto, este estudo objetivou revisar a literatura sobre a $\mathrm{HMI}$ objetivando proporcionar ao cirurgião-dentista que realiza atendimento infantil informações sobre o diagnóstico, as características clínicas e o tratamento.

\section{METODOLOGIA}

Revisão integrativa da literatura, sendo utilizado as bases de dados LILACS (Literatura Latino-Americana e do Caribe em Ciências da Saúde), PubMed/MEDLINE (U.S. National Library of Medicine), Web of Science (Clarivate Analytics) e Scopus Elsevier. Foram usadas as palavras-chaves "Hipoplasia do Esmalte Dentário/Dental Enamel Hypoplasia" e "Hipomineralização Molar-Incisivo/Molar Incisor Hypomineralization" e o operador booleano "AND".

A busca bibliográfica foi realizada por dois pesquisadores (LF e ICCL) que coletaram de forma independente as informações. Foram incluídos estudos observacionais (transversal, caso controle e coorte) e ensaios clínicos.

\section{REVISÃO DE LITERATURA}

\section{PREVALÊNCIA E DISTRIBUIÇÃO}

Diante das várias publicações sobre a temática, pode-se encontrar prevalências variadas de $\mathrm{HMI}$ nas diferentes populações, como $0,48 \%$ reportada em crianças indianas de 7 a 9 anos (SUBRAMANIAM; GUPTA; SHARMA, 2016) e $37,3 \%$ em estudo conduzido com crianças dinamarquesas de 6 a 8 anos (WOGELIUS; HAUBEK; POULSEN, 2008).

Negre-Barber et al. (2018), em estudo transversal realizado na Espanha com 414 crianças com idades entre 8 e 9 anos verificaram que 24,2\% apresentavam hipomineralização molar-incisivo. Destas, $72 \%$ apresentavam lesões leves e $28 \%$ lesões graves. A prevalência de cárie dentária foi maior entre as crianças com a forma grave $(60,7 \%)$ quando comparada àquelas crianças com a forma leve da doença $(43,1 \%)$ ou sem a presença de lesões hipomineralizadas $(45,5 \%)$.

Buchgraber, Kqiku e Ebeleseder (2017) em pesquisa sobre proporção e severidade da HMI na Áustria, encontraram uma prevalência de 7\%. Dos indivíduos afetados, $71 \%$ apresentavam opacidades demarcadas, $18 \%$ possuíam restaurações atípicas e $11 \%$ portavam fratura de esmalte pós-eruptiva. Foram localizadas lesões hipomineralizadas em outros dentes como canino e molar decíduos e canino permanente e pré-molares.

No Brasil, o estudo desenvolvido por Tourino et al. (2016), realizado com 1.181 escolares de 8 e 9 anos de idade, revelou uma prevalência de HMI de $20,4 \%$, sendo esta frequência considerada alta pelos autores. A HMI esteve associada à presença de cárie dentária, de defeitos de desenvolvimento de esmalte em molares decíduos e a experiência de asma/bronquite nos primeiros anos de vida.

Subramaniam, Gupta e Sharma (2016) ao examinarem 2.500 crianças indianas de 7 a 9 anos de idade, não 
encontraram predileção por sexo pela HMI. Nessa pesquisa, foram encontradas $0,48 \%$ das crianças com a condição, observando-se 68 dentes hipomineralizados e metade dos indivíduos com todos os quatro primeiros molares permanentes afetados. Dentre os molares permanentes, os inferiores $(29,4 \%)$ foram mais frequentemente afetados do que os molares superiores $(27,9 \%)$.

Relacionando a $\mathrm{HMI}$ e a qualidade de vida, Dantas-Neta et al. (2016) afirmaram em estudo brasileiro, desenvolvido no município de Teresina-PI, que escolares com HMI grave tinham maior impacto negativo sobre os sintomas e limitação funcional oral do que aqueles sem a condição, de acordo com a percepção dos pais ou responsáveis. O questionário de qualidade de vida, $\mathrm{CPQ}_{11-14}$ ISF:16 em sua versão brasileira foi usado para determinar o impacto das condições bucais no cotidiano nos indivíduos. A prevalência de $\mathrm{HMI}$ encontrada foi de $18,3 \%$.

No estudo conduzido na Índia por Mittal (2016), foi relatado uma ocorrência de $9,8 \%$ de $\mathrm{HMI}$ e maior proporção de indivíduos apresentando lesões de hipomineralização envolvendo ao mesmo tempo o primeiro molar permanente e incisivos permanentes $(31,1 \%)$, seguido de sujeitos com apenas os primeiros molares permanentes afetados $(27,6 \%)$. Com relação as faces do elemento dentário, encontrou-se que a face vestibular foi a mais afetada pela $\mathrm{HMI}$ e a lingual foi aquela que esteve mais livre da condição.

\section{ETIOLOGIA E FATORES ASSOCIADOS}

A etiologia da Hipomineralização Molar-Incisivo está relacionada a complicações durante o período de mineralização dos primeiros molares e incisivos permanentes (TOURINO et al., 2016). Essa fase se inicia no período de gestação e é finalizada ao longo dos três primeiros anos de vida (SALEM; AZIZ; ASADI, 2016). Assim, as anormalidades dentro deste período podem estar relacionadas à ocorrência de HMI (BEENTJES; WEERHEIJM; GROEN, 2002; TOURINO et al., 2016).

Fatores ligados as complicações pré, peri e pós-natais tem sido evidenciados (FAGRELL et al., 2011; SALEM; AZIZ; ASADI, 2016; WHATLING; FEARNE, 2008) e muitas condições de risco são relacionadas, incluindo problemas de gestação (SOUZA et al., 2012), parto prematuro e baixo peso ao nascer (ARROW, 2009; BROGARDH-ROTH; MATSSON; KLINGBERG, 2011; GHANIM et al., 2013); doenças da primeira infância como varíola (WHATLING; FEARNE, 2008), asma (GUERGOLETTE et al., 2009), otite média, infecções do trato urinário, amigdalite, febre alta (GHANIM et al., 2013), dermatite atópica, alergias alimentares (HERNANDEZ et al., 2018), distúrbios gastrointestinais e uso frequente de antibióticos (JEREMIAS et al., 2013, 2016; KÜHNISCH et al., 2014; SERNA et al., 2016; WRIGHT; CARRION; MORRIS, 2015).

A ocorrência de efeitos tóxicos ambientais nos dentes em desenvolvimento foi estudada na Finlândia e os resultados sugeriram que as crianças expostas a quantidades mais elevadas de dioxinas por meio do leite materno apresentaram mais frequentemente $\mathrm{HMI}$ quando comparadas às crianças que foram expostas a níveis menores (ALALUUSUA et al., 1996; FTEITA; ALI; ALALUUSUA, 2006). De modo semelhante, um outro estudo também cita as dioxinas armazenadas cronicamente no tecido adiposo da mãe e transmitidas para os lactentes como fator associado (SADASHIVAMURTHY; DESHMUKH, 2012).

Considerando-se que as células formadoras do esmalte são geneticamente controladas (WRIGHT; CARRION; MORRIS, 2015), a susceptibilidade genética também pode estar associada com a origem da HMI (JEREMIAS et al., 2013, 2016; KÜHNISCH et al., 2014; TEIXEIRA et al., 2017; VIEIRA; KUP, 2016). No estudo realizado por kühnisch et al. (2014) uma associação foi observada entre variações nos genes AMBN (Ameloblastin), ENAM (Enamelin), TUFT1 (Tuftelin 1), TFIP11 (Tuftelin interacting protein 11) e SCUBE 1 (Signal peptide, CUB domain and EGF like domain containing 1) e uma maior prevalência da condição. No entanto, os fatores ambientais que atuam durante a formação do esmalte também podem interferir na função das proteínas expressas por esses genes (SERNA et al., 2016; SILVA et al., 2016; TEIXEIRA et al., 2017).

\section{CARACTERÍSTICAS CLÍNICAS E CONSEQUÊNCIAS DA $\mathrm{HMI}$}

Os dentes hipomineralizados apresentam inicialmente lesões opacas bem delimitadas. 0 esmalte defeituoso tem espessura normal, superfície lisa e sua coloração pode variar entre branco, amarelo e castanho (WEERHEIJM et al., 2003). Com o aumento da severidade, pode ocorrer a perda do esmalte de superfície após a erupção do dente (WEERHEIJM et al., 2003).

As cavidades encontradas não estão em conformidade com o quadro de cárie dentária (WEERHEIJM et al., 2003). Na maioria dos molares permanentes, elas se apresentam de forma atípica com extensões para superfícies lisas como vestibular e lingual (WEERHEIJM et al., 2003). No limite entre dente e cavidade encontra-se com frequência áreas de opacidade (WEERHEIJM et al., 2003).

Em casos de extensa destruição coronária devido à $\mathrm{HMI}$ os elementos afetados podem ter indicação de exodontia (AHMADI; RAMAZANI; NOURINASAB, 2012). Dentes extraídos em consequência da hipomineralização podem ser identificados a partir da presença de opacidades demarcadas ou restaurações atípicas em outros primeiros molares permanentes combinados com a ausência de primeiro molar permanente (WEERHEIJM et al., 2003). É muito pouco provável a indicação de exodontia de incisivos permanentes hipomineralizados (WEERHEIJM et al., 2003).

Geralmente, as lesões nos incisivos manifestam-se na face vestibular como opacidades demarcadas e frequentemente não exibem fraturas (JEREMIAS et al., 2013), diferente do que acontece com os molares que se encontram sob influência direta das forças mastigatórias 
(JEREMIAS et al., 2013). Embora a HMI seja um defeito assimétrico, quando existe uma lesão grave em um dente é comum que o elemento contralateral também esteja envolvido (ALALUUSUA et al., 1996; JEREMIAS et al., 2003; WEERHEIJM, 2003).

As fraturas pós-eruptivas associadas a menor qualidade do esmalte promovem a formação de cavidades incomuns, o que facilita o acúmulo de biofilme nessas áreas e aumenta a susceptibilidade dos dentes com a $\mathrm{HMI}$ à cárie dentária (AHMADI; RAMAZANI; NOURINASAB, 2012; COSTA-SILVA et al., 2010; LYGIDAKIS et al., 2010; WEERHEIJM, 2003). Essas lesões cariosas podem mascarar as superfícies hipomineralizadas (MITTAL et al., 2016) conduzindo a rápida destruição da coroa dentária e a necessidade de restaurações atípicas (WEERHEIJM et al., 2003).

Adicionalmente, dentes hipomineralizados são mais propensos à ocorrência de hipersensibilidade e, por isso, a escovação dentária e o tratamento odontológico podem se tornar dificultados (AMERICANO et al., 2017; BUCHGRABER; KQIKU; EBELESEDER, 2017; WEERHEIJM et al., 2003). Portanto, a terapêutica a ser adotada pode ser complexa devido à dificuldade na obtenção de anestesia local e na adesão dos materiais restauradores ao esmalte deficiente (JASULAITYTE; VEERKAMP; WEERHEIJM, 2007; SALEM; AZIZ; ASADI, 2016; WEERHEIJM, 2004).

\section{DIAGNÓSTICO CLÍNICO COM FINALIDADE EPIDEMIOLÓGICA}

Em 2003, a Academia Europeia de Odontopediatria (EAPD) estabeleceu os critérios de diagnóstico para a $\mathrm{HMI}$ em estudos epidemiológicos (YANNAM; AMARLAL; REKHA, 2016). O 6o Congresso da EAPD foi voltado para a questão dos defeitos de mineralização envolvendo os primeiros molares permanentes (WEERHEIJM, 2003; WEERHEIJM et al., 2003). Concluiu-se que havia um número limitado de pesquisas que continham dados sobre a prevalência da HMl e que nesses estudos diferentes critérios de diagnóstico foram utilizados dificultando a comparação entre eles (WEERHEIJM, 2003; WEERHEIJM et al., 2003).

Discutiu-se que para o estabelecimento de um diagnóstico preciso da condição seria necessário a elaboração de um índice de pontuação simples e reprodutível que reduzisse as limitações encontradas nos métodos até então usados (WEERHEIJM et al., 2003). O Índice Modificado de Defeitos de Desenvolvimento do Esmalte (FDI, 1992), empregado em diversos trabalhos (BIOND et al., 2011; JÄLEVIK et al., 2001; RODRIGUES et al., 2015) foi considerado inadequado para uso nos estudos epidemiológicos, pois não leva em consideração a desagregação do esmalte após a erupção, um sinal comumente presente na HMI (WEERHEIJM et al., 2003).

Assim sendo, o grupo de trabalho da Academia Europeia de Odontopediatria definiu as características da condição e afirmou que todos os primeiros molares e incisivos permanentes devem ser examinados, cons- tituindo 12 dentes índices (WEERHEIJM et al., 2003). O exame físico intrabucal deve ser realizado após a profilaxia com os dentes úmidos e tem por base a idade de 8 anos (WEERHEIJM et al., 2003). Logo, a presença de um único primeiro molar permanente hipomineralizado já é suficiente para considerar o indivíduo portador da HMI.

De acordo com os critérios estabelecidos, cada elemento dentário deve ser classificado para: ausência ou presença de opacidades demarcadas (branco, amarelo ou castanho), fratura de esmalte pós-eruptiva, restauração atípica, extração de molar devido à HMI e falha de erupção de molar ou incisivo (WEERHEIJM et al., 2003; HERNANDEZ; BOJI; ESPASA, 2016; YANNAM; AMARLAL; REKHA, 2016). Com relação a severidade, as opacidades demarcadas são consideradas defeitos leves de HMI e fraturas pós-eruptivas, restaurações atípicas e extração de molar são considerados lesões graves (HANAN et al., 2015; JASULAITYTE; VEERKAMP; WEERHEIJM, 2007; JEREMIAS et al., 2013).

É necessário estabelecer critérios para o diagnóstico diferencial entre a HMI e condições semelhantes como: fluorose dentária, amelogênese imperfeita, hipoplasia do esmalte e cárie dentária. Na fluorose as opacidades são difusas e estão presentes em dentes homólogos (DANTAS-NETA et al., 2016; TEIXEIRA et al., 2017). A amelogênese imperfeita é uma alteração genética que pode envolver todos os dentes e estar associada a taurodontia e a mordida aberta anterior (GHANIM et al., 2015). Fraturas de esmalte pós-eruptivas devido à $\mathrm{HMI}$ apresentam bordas irregulares, ao contrário de quebras relacionadas à hipoplasia que têm arestas lisas e arredondadas (GHANIM et al., 2015; TEIXEIRA et al., 2017). Por fim, as lesões de cárie dentária aparecem como manchas brancas, opacas e irregulares localizadas em áreas de acúmulo de biofilme (GHANIM et al., 2015; TEIXEIRA et al., 2017).

\section{TRATAMENTO}

O tratamento das opacidades demarcadas pode ser realizado por meio de diferentes condutas dentre as quais o clareamento dentário (HARIKA et al., 2016) e mais recentemente a técnica de infiltração profunda (GIANNETTI et al., 2018), com objetivo de melhoria estética (GIANNETTI et al., 2018; HARIKA et al., 2016). Esta técnica se refere à abrasão mecânica inicial da superfície externa do esmalte, seguida por uma fase de erosão química com ácido clorídrico a $15 \%$, penetração através da porosidade do esmalte por resina fluida e, quando necessário, reparo da camada fina de esmalte perdido usando um compósito (GIANNETTI et al., 2018).

Em molares hipomineralizados, a aplicação direta de selantes apresenta taxa de sobrevivência adequada, sugerindo que podem ser utilizados para prevenir lesões cariosas (FRAGELLI et al., 2017).

Com relação à hipersensibilidade, o estudo de Pasini et al. (2018) que avaliou a sensibilidade dentária em crianças com dentes com MIH, concluiu que o uso 
do agente remineralizante contendo fosfopeptídeo de caseína-fosfato de cálcio amorfo (CPP-ACP) resultou em uma melhora significativa na sensibilidade dentária quando comparad ao creme dental convencional com flúor. Resultado semelhante também foi encontrado no estudo conduzido por Özgül et al. (2013), no qual foi avaliado o efeito de substâncias remineralizantes como: flúor, CPP-ACP e CPP-ACP adicionado flúor. Todos os métodos testados apresentaram redução significativa da hipersensibilidade em dentes com HMI após 3 meses de acompanhamento clínico (ÖZGÜL et al., 2013).

O uso de dessensibilizantes também é descrito na literatura. $O$ estudo desenvolvido por Bekes et al. (2017) revelou redução da hipersensibilidade em molares afetados pela HMI através da aplicação de pasta dessensibilizante contendo $8 \%$ de arginina e carbonato de cálcio associada a um programa de escovação com creme dental de mesma composição com adicional de 1450 ppm fluoreto e enxaguante bucal durante 8 semanas. Resultados positivos de redução de sensibilidade foram relatados imediatamente e após a finalização do estudo (BEKES et al., 2017).

Para os dentes afetados pela HMI e cavitados, alguns estudos indicam a realização de restaurações temporárias com Cimento de lonômero de Vidro (CIV) restaurador e seu acompanhamento clínico e radiográfico (FRAGELLI et al., 2015; GROSSI et al., 2018; OLIVEIRA; FAVRETTO; CUNHA, 2015; ORELLANA; PÉREZ, 2017). Nos trabalhos de Grossi et al. (2018) e Fragelli et al. (2015) que analisaram restaurações com CIV em dentes hipomineralizados, a taxa de sucesso do tratamento após 12 meses de supervisão foi, respectivamente, de $98,3 \%$ e $78 \%$. Em ambos os estudos, o preparo da estrutura dentária se limitou à remoção de áreas afetadas pela cárie dentária, preservando a área com hipomineralização quando esta se apresentou saudável. A aplicação de verniz fluoretado associada ao preenchimento de cavidades com CIV é uma medida que auxilia no fortalecimento da estrutura dentária (OLIVEIRA; FAVRETTO; CUNHA, 2015).

As restaurações diretas têm sua indicação quanto mais conservador for o preparo do dente (SOUZA et al., 2017). Em dentes com grandes extensões de destruição coronária, restaurações indiretas como onlays (DHAREULA et al., 2018) e coroas de aço (HARIKA et al., 2016) podem ser utilizadas como tratamento para os dentes afetados. Nos casos de extensa destruição coronária, a exodontia pode ser indicada juntamente com o tratamento ortodôntico para reabilitação do arco funcional (MATHU-MUJU; KENNEDY, 2016).

\section{DISCUSSÃO}

O primeiro estudo epidemiológico sobre hipomineralização em primeiros molares e incisivos permanentes data de 1987, sendo desenvolvido na Suécia (KOCH et al., 1987), porém, apenas na última década, os estudos vêm sendo impulsionados com a finalidade de ampliar o nível de conhecimento sobre a doença (BUCHGRABER; KQIKU; EBELESEDER, 2017). Pode-se inferir que esse crescente interesse no âmbito científico ocorreu pela semelhança clínica da HMI com a cárie dentária e a maior susceptibilidade a lesões cariosas nos dentes afetados pela condição (AHMADI; RAMAZANI; NOURINASAB, 2012; COSTA-SILVA et al., 2010; LYGIDAKIS et al., 2010; WEERHEIJM, 2003), o que afeta de forma negativa a saúde bucal das crianças (DANTAS-NETA et al., 2016). Assim, é comum encontrarmos crianças que tenham molares hipomineralizados associados à cárie dentária.

Atualmente, não existe um padrão estabelecido de prevalência da $\mathrm{HMI}$ nas populações (ANDRADE et al., 2017; BUCHGRABER; KQIKU; EBELESEDER, 2017; ZHAO et al., 2017) e, portanto, é imprescindível que pesquisas voltadas para o tema sejam estimuladas. O conhecimento da prevalência é útil na verificação de alterações de saúde ao longo do tempo e no planejamento de políticas de saúde, uma vez que permite organizar os recursos de saúde existentes para as condições mais importantes. Essa variabilidade pode ocorrer, dentre outros fatores, devido às diferenças metodológicas encontradas entre os estudos (BHASKAR; HEGDE, 2014; CROMBIE; MANTON; KILPATRICK, 2009; JÄLEVIK, 2010; WEERHEIJM et al., 2003; WILLIAM; MESSER; BURROW, 2006; WILLMOTT, 2011), que podem ser paulatinamente reduzidas com o uso crescente do índice proposto pela EAPD. Atualmente, este método é o mais abordado entre os estudos e mais consolidado na literatura (ANDRADE et al., 2017; BUCHGRABER; KQIKU; EBELESEDER, 2017; SALEM; AZIZ; ASADI, 2016; TEIXEIRA et al., 2017).

O índice EAPD surgiu devido a necessidade de realizar estudos que pudessem ser comparados com o objetivo de estabelecer dados de prevalência representativos da população estudada e estratégias de diagnóstico e tratamento. A idade de 8 anos foi escolhida como sendo a ideal pois considera-se que na maioria dos indivíduos os molares e incisivos permanentes já estão erupcionados e há menor risco das lesões hipomineralizadas serem mascaradas pela cárie dentária, pois nesse momento os dentes apresentam curto período em boca havendo pouco tempo para a manifestação de morbidades.

Apesar de ser amplamente utilizado, o índice EAPD ainda apresenta limitações, como o não estabelecimento do diâmetro mínimo a partir do qual deve ser considerado como lesão hipomineralizada. A Federação Internacional de Odontologia (FDI,1992) recomenda que apenas os defeitos maiores que $2 \mathrm{~mm}$ devem ser registrado e, portanto, esse parâmetro é utilizado em alguns estudos (HANAN et al., 2015; JEREMIAS et al., 2013; RODRIGUES et al., 2015). Além disso, a Academia Europeia de Odontopediatria requer a realização do exame clínico após a profilaxia, fato que não se apresenta viável em alguns contextos dos estudos, pois são realizados em ambiente escolar (JEREMIAS et al., 2013; MITTAL et al., 2016; SUBRAMANIAM; GUPTA; SHARMA, 2016).

Com relação à etiologia, a maioria dos estudos corre- 
laciona fatores ambientais, genéticos, pré-natais, perinatais e pós-natais (ARROW, 2009; BEENTJES; WEERHEIJM; GROEN, 2002; BROGARDH-ROTH; MATSSON; KLINGBERG, 2011; GHANIM et al., 2013; GUERGOLETTE et al., 2009; JÄLEVIK et al., 2001; PITIPHAT et al., 2014; WHATLING; FEARNE, 2008), porém, os achados são inconclusivos. Sabe-se que a sua origem está relacionada a anormalidades que ocorreram entre a gestação e os três primeiros anos de vida da criança (SALEM; AZIZ; ASADI, 2016), pois este é o momento referente ao período de odontogênese dos incisivos e molares. Apesar disso, existe uma dificuldade de se estabelecer um agente patológico particular e os fatores associados à etiologia da $\mathrm{HMI}$ devido à maioria dos estudos serem baseados na lembrança dos pais (WUOLLET et al., 2016). Isto se constitui em um viés de memória importante que dificulta a elaboração de hipóteses. Para minimizar essa questão, sugere-se a realização de estudos longitudinais prospectivos (HOCHMAN et al., 2005), nos quais o acompanhamento dos casos permite a coleta das informações sobre a etiologia no momento exato da ocorrência reduzindo a influência de erros e fatores de confusão no estudo.

O conhecimento dos fatores etiológicos pode contribuir para a identificação de crianças que são mais propensas a esta condição, bem como o estabelecimento de medidas preventivas, com o objetivo de evitar ou reduzir a possibilidade de manifestação das consequências desse defeito de esmalte. Nesse sentido, o aconselhamento e acompanhamento médico e odontológico pré, peri e pós-natais devem ser incentivados.

Devido à influência das forças mastigatórias, o esmalte dos molares permanentes pode quebrar com maior facilidade expondo a dentina ao meio bucal (JÄLEVIK et al., 2001; JEREMIAS et al., 2013). Desse modo, as fraturas de esmalte exibem áreas de maior retenção de biofilme e, devido à sensibilidade aumentada pela própria condição e exposição dos túbulos dentinários, há dificuldade de escovação e manutenção da higiene bucal, contribuindo para o aparecimento de lesões cariosas com maior facilidade. Além disso, quando os incisivos são afetados, os pacientes reportam problemas estéticos (JEREMIAS et al., 2013; TOURINO et al., 2016; WEERHEIJM, 2003), a depender da severidade das lesões encontradas.

Deste modo, portadores de $\mathrm{HMI}$ apresentam maior necessidade de intervenções clínicas que objetivam de reduzir ou eliminar as consequências deste gravo, como a cárie dentária, a degradação extensa do esmalte e a indicação de exodontia. São pacientes que exigirão consultas odontológicas de retorno com intervalos de tempo mais curtos para acompanhamento e realização de medidas terapêuticas individuais.

Face a esses aspectos, a qualidade de vida dessas crianças pode ser afetada, podendo ter aumentados 0 risco de desenvolver medo, ansiedade e problemas de comportamento no ambiente do consultório odontológico (ALLAZZAM; ALAKI; EL MELIGY, 2014; JÄLEVIK; KLINGBERG, 2012; JASULAITYTE; VEERKAMP; WEERHEIJM, 2007; KOS-
MA et al., 2016) devido às dificuldades na realização dos procedimentos, na adesão das crianças ao tratamento e na colaboração da manutenção de uma adequada higiene bucal.

O tratamento odontológico a ser realizado estará na dependência da gravidade das lesões presentes (STOJKOVIC et al., 2017). As lesões leves, no geral, recebem medidas profiláticas e à proporção que a gravidade aumenta, tratamentos mais invasivos são necessários. Não existem protocolos clínicos terapêuticos padronizados de acordo com a severidade da lesão hipomineralizada, visto que a terapêutica envolve principalmente a redução e controle das consequências da HMl e não age diretamente na sua etiologia.

\section{CONCLUSÃO}

A Hipomineralização Molar-Incisivo é uma importante condição que deve receber a adequada atenção por parte do cirurgião-dentista em função das consequências clínicas e estéticas que acarreta ao paciente, interferindo negativamente na qualidade de vida da criança nos casos mais graves. Portanto, novas pesquisas são necessárias com a finalidade de expandir o conhecimento teórico e conceitual acerca da HMI, bem como reunir evidências para que os cirurgiões-dentistas que prestam atendimento infantil estejam aptos a ofertar ao paciente o correto diagnóstico e a melhor opção terapêutica.

\section{CONFLITO DE INTERESSES}

Não existem conflitos de interesse.

\section{REFERÊNCIAS}

AHMADI, R.; RAMAZANI, N.; NOURINASAB, R. Molar incisor hypomineralization: a study of prevalence and etiology in a group of Iranian children. Iran. J. Pediatr., Tehran, v. 22, n. 2, p. 245-251, June 2012.

ALALUUSUA, S. et al. Developmental dental defects associated with long breast feeding. Eur. J. Oral Sci., Copenhagen, v. 104, n. 5-6, p. 493-497, Oct./Dec. 1996.

ALLAZZAM, S.M.; ALAKI, S.M.; EL MELIGY, O.A.S. Molar incisor hypomineralization, prevalence, and etiology. Int. J. Dent., Cairo, v. 2014, n. 234508, p.8, May 2014.

AMERICANO, G. C. et al. A systematic review on the association between molar incisor hypomineralization and dental caries. Int. J. Paediatr. Dent. Oxford, v. 27, n. 1, p. 11-21, Jan. 2017.

ANDRADE, N.S. et al. Molar incisor hypomineralization in HIV-infected children and adolescents. Spec. Care Dentist., Chicago, v. 37, n.1, p. 28-37, Jan. 2017.

ARROW, P. Risk factors in the occurrence of enamel defects of the first permanent molars among schoolchildren in Western Australia. Community Dent. Oral Epidemiol., Copenhagen, v. 37, n. 5, p. 405-415, Oct. 2009.

BEENTJES, V.E.; WEERHEIJM, K.L.; GROEN, H.J. Factors involved in the aetiology of molar-incisor hypomineralisation (MIH). Eur. J. Paediatr. Dent., Milano, v. 3, n. 1, p. 9-13, Mar. 2002.

BEKES, K. et al. Efficacy of desensitizing products containing $8 \%$ argi- 
nine and calcium carbonate for hypersensitivity relief in $\mathrm{MIH}$-affected molars: an 8-week clinical study. Clin. Oral Investig., Berlin, v. 21, n. 7, p. 2311-2317, Sept. 2017.

BHASKAR, S.A.; HEGDE, S. Molar-incisor hypomineralization: Prevalence, severity and clinical characteristics in 8 to 13 year-old children of Udaipur, India. J. Indian Soc. Pedod. Prev. Dent., Chandigarh, v. 32, n. 4, p. 322-329, Oct./Dec. 2014.

BIONDI, A.M. et al. Prevalence of molar incisor hypomineralization in the city of Buenos Aires. Acta Odontol. Latinoam., Buenos Aires, v. 24, n. 1, p. 81-85, Apr. 2011.

BROGARDH-ROTH, S.; MATSSON, L.; KLINGBERG, G. Molar-incisor hypomineralization and oral hygiene in $10-$ to -12 -yr-old Swedish children born preterm. Eur. J. Oral Sci., Copenhagen, v. 119, n. 1, p. 33-39, Feb. 2011.

BUCHGRABER, B.; KQIKU, L.; EBELESEDER, K.A. Molar incisor hypomineralization: proportion and severity in primary public school children in Graz, Austria. Clin. Oral Investig., Berlin, v. 22, n. 2, p. 757-762, Mar. 2017.

CLARKSON, J. A review of the developmental defects of enamel index (DDE). Int. Dent. J., London, v. 42, n. 5, p. 411-426, Dec. 1992.

COSTA-SILVA, C.M. et al. Molar incisor hypomineralization: prevalence, severity and clinical consequences in Brazilian children. Int. J. Paediatr. Dent., Oxford, v. 20, n. 6, p. 426-434, Nov. 2010.

CROMBIE, F.; MANTON, D.; KILPATRICK, N. Aetiology of molarincisor hypomineralization: A critical review. Int J Paediatr Dent, Oxford, v. 19, n. 2, p. 73-78, Mar. 2009.

DANTAS-NETA, N.B. et al. Impact of molar-incisor hypomineralization on oral health-related quality of life in schoolchildren. Braz. Oral Res., São Paulo, v. 30, n. 1, e117, Oct. 2016.

DHAREULA, A. et al. Esthetic rehabilitation of first permanent molars affected with severe form of Molar Incisor Hypomineralization using indirect composite onlays-A case series. Pediatr. Dent. J., Tokyo, v. 28, n. 2, p. 62-67, Aug. 2018.

ELFRINK, M.E.C. et al. Deciduous molar hypomineralization and molar incisor hypomineralization. J. Dent. Res., Chicago, v. 91, n. 6, p. 551555, Jun. 2012.

FAGRELL, T.G. et al. Aetiology of severe demarcated enamel opacities - an evaluation based on prospective medical and social data from 17.000 children. Swed. Dent. J., Jönköping, v. 35, n. 2, p. 57-67, 2011.

FÉDÉRATION DENTAIRE INTERNATIONALE (FDI). Comission on Oral health, Research and Epidemiology. A review of the developmental defects index (DDE Index). Int. Dent. J., London, v. 42, n. 6, p. 411-426, Dec. 1992.

FORD, D. et al. A controlled study of risk factors for enamel hypoplasia in the permanent dentition. Pediatr. Dent., Chicago, v. 31, n. 5, p. 382388, Sep./Oct. 2009.

FRAGELLI, C.M. et al. Molar incisor hypomineralization (MIH): conservative treatment management to restore affected teeth. Braz. Oral Res., São Paulo, v. 29, n. 1, p. 1-7, June 2015.

FRAGELLI, C. M. B. et al. Survival of sealants in molars affected by molar-incisor hypomineralization: 18-month follow-up. Braz. Oral Res., São Paulo, v. 31, n. 1, p. 1-9, Apr. 2017.
FTEITA, D., ALI, A., ALALUUSUA, S. Molar-incisor hypomineralization (MIH) in a group of school-aged children in Benghazi, Libya. Eur. Arch. Paediatr. Dent., Leeds, v. 7, n. 2, p. 92-95, June 2006.

GHANIM, A. et al. A practical method for use in epidemiological studies on enamel hypomineralisation. Eur. Arch. Paediatr. Dent., Leeds, v. 16, n. 3, p. 235-246, June 2015.

GHANIM, A. et al. Risk factors in the occurrence of molar-incisor hypomineralization amongst a group of Iraqi children. Int. J. Paediatr. Dent., Oxford, v. 23, n. 3, p. 197-206, Mar. 2013.

GIANNETTI, L. et al. Deep infiltration for the treatment of hypomineralized enamel lesions in a patient with molar incisor hypomineralization: a clinical case. J. Biol. Regul. Homeost. Agents., Milano, v. 32, n. 3, p. 751-754, May/June 2018.

GROSSI, J. A. et al. Glass hybrid restorations as an alternative for restoring hypomineralized molars in the ART model. BMC Oral Health., London, v. 18, n. 1, p. 65-72, Apr. 2018.

GUERGoletTE, R. P. et al. Prevalence of developmental defects of enamel in children and adolescents with asthma. J. Bras. Pneumol., Brasilia, v. 35, n. 9, p. 295-300, Apr. 2009.

HANAN, S.A. et al. Molar-incisor hypomineralization in schoolchildren of Manaus, Brazil. Pesqui. Bras. Odontopediatr. Clin. Integr., João Pessoa, v. 15, n. 1, p. 309-317, 2015.

HARIKA, R. et al. A novel clinical approach for long-term retention and durability of resin restorations bonded to multiple developmental defects of enamel. J. Int. Soc. Prev. Community Dent., Mumbai, v. 6, n. 6, p. 597-601, Nov./Dec. 2016.

HERNANDEZ, M. et al. Molar-Incisor Hypomineralization: Positive Correlation with Atopic Dermatitis and Food Allergies. J. Clin. Pediatr. Dent., Birmingham, v. 42, n. 5, p. 1-5, May, 2018.

HERNANDEZ, M.; BOJI, J.R.; ESPASA, E. Do We Really Know the Prevalence of MIH? J. Clin. Pediatr. Dent., Birmingham, v. 40, n. 4, p. 259-263, 2016.

HOCHMAN, B. et al. Research designs. Acta Cir. Bras., São Paulo, v. 20, n. 1, supl. 2, p. 2-9, 2005.

JÄLEVIK, B. et al. The prevalence of demarcated opacities in permanent first molars in a group of Swedish children. Acta Odontol. Scand., Stockholm, v. 59, n. 5, p. 255-260, Oct. 2001.

JÄLEVIK, B. Prevalence and diagnosis of Molar-Incisor Hypomineralisation (MIH): A systematic review. Eur Arch Paediatr Dent, Leeds, v. 11, n. 2, p. 59-64, Apr. 2010.

JÄLEVIK, B.; KLINGBERG, G. Treatment outcomes and dental anxiety in 18-year-olds with $\mathrm{MIH}$, comparisons with healthy controls: a longitudinal study. Int. J. Paediatr. Dent., Oxford, v. 22, n. 2, p. 85-91, Mar. 2012.

JASULAITYTE, L.; VEERKAMP, J.S.; WEERHEIJM, K.L. Molar incisor hypomineralization: review and prevalence data from the study of primary school children in Kaunas/Lithuania. Eur. Arch. Paediatr. Dent., Leeds, v. 8, n. 2, p. 87-94, June 2007.

JEREMIAS, F. et al. Dental caries experience and molar-incisor hypomineralization. Acta Odontol. Scand., Stockholm, v. 71, n. 3-4, p. 870-876, May/July 2013.

JEREMIAS, F. et al. Family-based genetic association for molar-incisor hypomineralization. Caries Res., Basel, v. 50, n. 3, p. 310-318, May, 2016.

$\mathrm{KOCH}, \mathrm{G}$. et al. Epidemiologic study of idiopathic enamel hypomineralization in permanent teeth of Swedish children. Community Dent Oral Epidemiol, Copenhagen, v. 15, n. 5, p. 279-285, Oct. 1987.

KOSMA, I. et al. Molar incisor hypomineralisation (MIH): correlation 
with dental caries and dental fear. Eur. Arch. Paediatr. Dent., Leeds, v. 17, n. 2, p. 123-129. Apr. 2016.

KÜHNISCH, J. et al. Genomewide association study (GWAS) for molar-incisor hypomineralization (MIH). Clin. Oral Investig., Berlin, v. 18, n. 2, p. 677-682, 2014.

LYGIDAKIS, N.A. et al. Best Clinical Practice Guidance for clinicians dealing with children presenting with Molar-Incisor-Hypomineralisation (MIH): An EAPD Policy Document. Eur. Arch. Paediatr. Dent., Leeds, v. 11, n. 2, p. 75-81, Apr. 2010.

MATHU-MUJU, K. R.; KENNEDY, D.B. Loss of permanent first molars in the mixed dentition: Circumstances resulting in extraction and requiring orthodontic management. Pediatr Dent., Chicago, v. 38, n. 5, p. 46-53, Oct. 2016.

MITTAL, N. Phenotypes of enamel hypomineralization and molar incisor hypomineralization in permanent dentition: Identification, quantification and proposal for classification. J. Clin. Pediatr. Dent., Birmingham, v. 40, n. 5, p. 367-374, 2016.

MITTAL, R. et al. Assessment of association between molar incisor hypomineralization and hypomineralized second primary molar. J. Int. Soc. Prev. Community Dent., Mumbai, v. 6, n. 1, p. 34-39, Jan./Feb. 2016.

NEGRE-BARBER, A. et al. Degree of severity of molar incisor hypomineralization and its relation to dental caries. Sci. Rep., London, v. 8, n. 1, p. 1248-1254, Jan. 2018.

OLIVEIRA, D.C.; FAVRETTO, C.O.; CUNHA, R.F. Molar incisor hypomineralization: Considerations about treatment in a controlled longitudinal case. J. Indian Soc. Pedod. Prevl Dent., Chandigarh, v. 33, n. 2, p. $152-$ 155, Apr./June 2015.

ORELLANA, C.; PÉREZ, V. Modified glass ionomer and orthodontic band: an interim alternative for the treatment of molar incisor hypomineralization. A case report. J. Oral Res., Concepción, v. 6, n. 3, p. 70-74, Mar. 2017.

ÖZGÜL, B.M. et al. Clinical evaluation of desensitizing treatment for incisor teeth affected by molar-incisor hypomineralization. J. Clin. Pediatr. Dent., Birmingham, v. 38, n. 2, p.101-105, 2013.

PASINI, M. et al. Molar incisor hypomineralization treatment with casein phosphopeptide and amorphous calcium phosphate in children. Minerva Stomatol., Torino, v. 67, n. 1, p. 20-25, Feb. 2018.

PITIPHAT, W. et al. Factors associated with molar incisor hypomineralization in Thai children. Eur. J. Oral Sci., Copenhagen, v. 122, n. 4, p. 265-70, Aug. 2014.

RODRIGUES, F.C.N. et al. Molar-Incisor hypomineralization in schoolchildren of Sao Luis, Brazil Maranhao: prevalence and associated factors. Pesqui. Bras. Odontopediatr. Clin. Integr., João Pessoa, v. 15, n. 1, p. 271-278, Aug. 2015.

SADASHIVAMURTHY, P.; DESHMUKH, S. Missing links of Molar Incisor Hypomineralization: A review. J. Int. Oral Health, Ahmedabad, v. 4, n. 1, p. 1-10, Jan. 2012.

SALEM, K.; AZIZ, D.; ASADI, M. Prevalence and predictors of Molar Incisor Hypomineralization (MIH) among rural children in Northern Iran. Iran. J. Public. Health, Tihrān, v. 45, n. 11, p. 1528-1530, Nov. 2016.

SEOW, W.K. Developmental defects of enamel and dentine: challenges for basic science research and clinical management. Aust. Dent. J., Sydney, v. 59, n. 1, suppl. 1, p. 143-154, June 2014.

SEOW, W.K. et al. Comparison of enamel defects in the primary and permanent dentitions of children from a low fluoride district of Australia. Pediatr. Dent., Chicago, v. 33, n. 3, p. 207-212, May/June 2011.
SERNA, C. et al. Drugs related to the etiology of molar incisor hypomineralization: a systematic review. J. Am. Dent. Assoc., Chicago, v. 147, n. 2, p. 120-130, Feb. 2016.

SILVA, M. J. et al. Etiology of molar incisor hypomineralization - a systematic review. Community Dent. Oral Epidemiol., Copenhagen, v. 44, n. 4, p. 342-353, Aug. 2016.

SOUZA, J. F. et al. Eighteen-month clinical performance of composite resin restorations with two different adhesive systems for molars affected by molar incisor hypomineralization. Clin Oral Investig., Berlin, v. 21, n. 5, p. 1725-1733, June 2017.

SOUZA, J.F. et al. Molar incisor hypomineralisation: possible aetiological factors in children from urban and rural areas. Eur. Arch. Paediatr. Dent., Leeds, v. 13, n. 4, p. 164-170, Aug. 2012.

STOJKOVIC, B. et al. Molar-incisor hypomineralization: therapeutic challenge to paediatric dentistry practice. Acta Stomatol. Naissi., Nis, v. 33, n. 75, p. 1718-1729, June 2017.

SUBRAMANIAM, P.; GUPTA, T.; SHARMA, A. Prevalence of molar incisor hypomineralization in 7-9-year-old children of Bengaluru City, India. Contemp. Clin. Dent., Mumbai, v. 7, n. 1, p. 11-15, Jan./Mar. 2016.

TEIXEIRA, R.J.P.B. et al. Exploring the association between genetic and environmental factors and molar incisor hypomineralization: evidence from a twin study. Int. J. Paediatr. Dent., Oxford, v. 28, n. 2, p. 198-206, Aug. 2017.

TOURINO, L. F. et al. Association between molar incisor hypomineralization in schoolchildren and both prenatal and postnatal factors: A Population based study. PLoS One, San Francisco, v. 11, n. 6, e0156332, June 2016.

VIEIRA, A.R.; KUP, E. On the etiology of molar-incisor hypomineralization. Caries Res. Basel, v. 50, n. 2, p. 166-169, May, 2016.

VILANI, P.N.L. et al. Hipomineralização molar-incisivo: relato de caso clínico. Rev. Faculdade Odontol. Lins, São Paulo, v. 23, n. 1, p. 64-68, jan./jun. 2014.

WEERHEIJM, K.L. et al. Judgement criteria for Molar Incisor Hypomineralisation $(\mathrm{MIH})$ in epidemiologic studies: a summary of the European meeting on MIH held in Athens, 2003. Eur. J. Peadiatr. Dent., Milano, v. 4, n. 3, p. 110-113, Sept. 2003.

WEERHEIJM, K.L. Molar Incisor Hypomineralisation (MIH). Eur. J. Paediatr. Dent., Milano, v. 4, n. 3, p. 114-120, Sept. 2003.

WEERHEIJM, K.L. Molar incisor hypomineralization (MIH): clinical presentation, aetiology and management. Dent. Update, London, v. 31, n. 1, p. 9-12, Jan./Feb. 2004.

WEERHEIJM, K.L.; JALEVIK, B.; ALALUUSUA, S. Molar-incisor hypomineralisation. Caries Res., Basel, v. 35, n. 5, p. 390-391, Sept./Oct. 2001.

WHATLING, R.; FEARNE, J.M. Molar incisor hypomineralization: a study of aetiological factors in a group of UK children. Int. J. Paediatr. Dent., Oxford, v. 18, n. 3, p. 155-162, May 2008.

WILLIAM, V.; MESSER, L.B.; BURROW, M.F. Molar-incisor hypomineralization: review and recommendations for clinical management. Pediatr. Dent., Chicago, v. 28, n. 3, p. 224-232, May/June 2006.

WILLMOTT, N. Molar incisor hypomineralization. Dent. Nurs, Fleetwood, v. 7, n. 3, p. 132-137, Mar. 2011.

WOGELIUS, P., HAUBEK, D., POULSEN, S. Prevalence and distribution of demarcated opacities in permanent 1st molars and incisors in 6 - to 8 -year-old Danish children. Acta Odont. Scand., Stockholm, v. 66, n. 1, p. 58-64, Feb. 2008. 
WRIGHT, J.T.; CARRION, I.A.; MORRIS, C.A. The molecular basis of hereditary enamel defects in humans. J. Dent. Res., Chicago, v. 94, n. 1, p. 52-61, Jan. 2015.

WUOLLET, E. et al. Molar-incisor hypomineralization and the association with childhood illnesses and antibiotics in a group of Finnish children. Acta Odontol. Scand., Stockholm, v. 74, n. 5, p. 416-422, July 2016.
YANNAM, S.D.; AMARLAL, D.; REKHA, C.V. Prevalence of molar incisor hypomineralization in school children aged 8-12 years in Chennai. J. Indian. Soc. Pedod. Prev. Dent., Chandigar, v. 34, n. 2, p. 134-138, Apr./June 2016.

ZHAO, D. et al. The prevalence of molar incisor hypomineralization: evidence from 70 studies. Int. J. Paediatr. Dent., Oxford, v. 28, n.2, p. 170-179, July 2017. 